\title{
Developing German Semantics on the basis of Parallel LFG Grammars
}

\author{
Sina Zarrieß \\ Department of Linguistics \\ University of Potsdam, Germany \\ sina@ling.uni-potsdam.de
}

\begin{abstract}
This paper reports on the development of a core semantics for German which was implemented on the basis of an English semantics that converts LFG f-structures to flat meaning representations in a Neo-Davidsonian style. Thanks to the parallel design of the broad-coverage LFG grammars written in the context of the ParGram project (Butt et al., 2002) and the general surface independence of LFG f-structure analyses, the development process was substantially facilitated. We also discuss the overall architecture of the semantic conversion system from a crosslinguistic, theoretical perspective.
\end{abstract}

\section{Introduction}

This paper reports on the development of a core semantics for German which was implemented on the basis of an English semantics that converts LFG f-structures to flat meaning representations in a Neo-Davidsonian style. The development strategy relies on the parallel design of the broad-coverage LFG grammars written in the context of the ParGram project (Butt et al., 2002). We will first describe the overall architecture of the semantic conversion system as well as the basic properties of the semantic representation. Section 3 discusses the development strategy and the core semantic phenomena covered by the German semantics. In section 3.4, we will discuss the benefits and the limitations of the presented architecture for crosslingual semantics by means of an example phenomenon, the semantics of clauseembedding verbs. The rest of this introduction will be devoted to the broader theoretical context of this work.

Recently, the state of the art in widecoverage parsing has made wide-coverage semantic processing come into the reach of research in computational semantics (Bos et al., 2004). This shift from the theoretical conception of semantic formalisms to wide-coverage semantic analysis raises many questions about appropriate meaning representations as well as engineering problems concerning the development and evaluation strategies of semantic processing systems. The general aim of this work is to explore wide-coverage LFG syntax as a backbone for linguistically motivated semantic processing.

Research in the framework of LFG has traditionally adopted a crosslingual perspective on linguistic theory (Bresnan, 2000). In the context of the ParGram project, a number of high quality, broad-coverage grammars for several languages have been produced over the years (Butt et al., 2002; Butt and King, 2007). ${ }^{1}$ The project's research methodology particularly focusses on parallelism which means that the researchers rely on a common syntactic theory as well as development tools, but which also concerns parallelism on the level of syntactic analyses. As the LFG formalism assumes a two-level syntax that di-

\footnotetext{
${ }^{1}$ Also see the webpage for a nice project overview: http://www2.parc.com/isl/groups/nltt/pargram/
} 
vides the analysis into a more language and surface dependent constituent structure and a functional structure which basically represents the surface independent grammatical relations of a sentence, it constitutes a particularly appropriate basis for large-scale, multilingual syntax.

Parallel grammar development bears the practical advantage that the resources developped for a particular language can often easily be ported to related languages. Kim et al. (2003) report that the Korean ParGram grammar was constructed in two months by adapting the Japanese grammar for Korean. Moreover, parallel grammars have a straightforward application in multilingual NLP tasks like machine translation (Frank, 1999).

A general motivation for multilingual, deep grammars are higher-level NLP tasks which involve some kind of semantic or meaningsensititive processing (Butt and King, 2007). The work presented in this paper shows that parallel grammar development not only facilitates porting of grammars, but substantially facilitates the development of resources and applications that involve such a parallel grammar. We rely on the semantic conversion system presented in (Crouch and King, 2006) to implement a system that derives semantic representations from LFG fstructures for German. Due to the parallelism of syntactic f-structure input, the German core semantics could be implemented within a single month.

\section{F-Structure Rewriting as an LFG Semantics}

Since the early days of LFG, there has been research on interfacing LFG syntax with various semantic formalisms (Dalrymple, 1999). For the English and Japanese ParGram grammar, a broad-coverage, glue semantic construction has been implemented by (Crouch, 1995; Umemoto, 2006). In contrast to these approaches, the semantic con- version described in (Crouch and King, 2006) is not driven by a specific semantic theory about meaning representation, nor by a theoretically motivated apparatus of meaning construction. Therefore, we will talk about "semantic conversion" instead of "construction" in this paper.

The main idea of the system is to convert the surface-independent, syntactic relations and features encoded in an f-structure to normalized semantic relations. The representation simplifies many phenomena usually discussed in the formal semantic literature (see the next section), but is tailored for use in Question Answering (Bobrow et al., 2007a) or Textual Entailment (Bobrow et al., 2007b) applications.

The semantic conversion was implemented by means of the XLE platform, used for grammar development in the ParGram project. It makes use of the built-in transfer module to convert LFG f-structures to semantic representations. The idea to use transfer rules to model a semantic concstruction has also been pursued by (Spreyer and Frank, 2005) who use the transfer module to model a RMRS semantic construction for the German treebank TIGER .

\subsection{The Semantic Representation}

As a first example, a simplified f-structure analysis for the following sentence and the corresponding semantic representation are given in figure 1.

(1) In the afternoon, John was seen in the park.

The basic idea of the representation exemplified in figure 1 is to represent the syntactic arguments and adjuncts of the main predicate in terms of semantic roles of the context introduced by the main predicate or some higher semantic operator. Thus, the grammatical roles of the main verb in sentence (1) are semantically normalized such that the subject of the passive becomes a theme and an unspecified agent is introduced, see figure 1 . The role of the modifiers are speci- 
fied in terms of their head preposition. This type of semantic representation is inspired by Neo-Davidsonian event semantics (Parsons, 1990). Other semantic properties of the event introduced by the main verb such as tense or nominal properties such as quantification and cardinality are explicitely encoded as conventionalized predications.

The contexts can be tought of as propositions or possible worlds. They are headed by an operator that can recursively embed further contexts. Context embeddings can be induced by lexical items or syntactic constructions and include the following operators: (i) negation (ii) sentential modifiers (possibly) (iii) coordination with or (iv) conditionals (v) some subordinating conjunctions (without) (vi) clause-embedding verbs (doubt).

The representation avoids many formal semantic complexities typically discussed in the literature, for instance the interpretation of quantifiers by encoding them as conventionalized semantic predications. Given this skolemized first-order language, the task of textual entailment can be conceived as matching the hypothesis representation against the semantic representation of the text where higher-order reasonning is approximated by explicit entailment rules (e.g. all entails some, past does not entail present), see (Bobrow et al., 2007b) for a presentation of an RTE system based on this semantic representation.

\subsection{The Semantic Conversion}

The XLE transfer module, which we use for the implementation of the conversion of $f-$ structures to semantic representations, is a term rewrite system that applies an ordered list of rewrite rules to a given f-structure input and yields, depending on the rewrite rules, new f-structures (e.g. translated fstructures) or semantic representations. The technical features of the XLE transfer module are described in (Crouch et al., 2006). An important feature for large-scale develop-
+VTYPE $(\% \mathrm{~V}, \% \%),+$ PASSIVE $(\% \mathrm{~V},+)$, OBL-AG (\%V, \%LogicalSUBJ), PTYPE (\%LogicalSUBJ , \% $)$, OBJ ( $\%$ LogicalSUBJ , \%P)

$==>\operatorname{SUBJ}(\% \mathrm{~V}, \% \mathrm{P}), \arg (\% \mathrm{~V}, \% \mathrm{~N}, \% \mathrm{P})$.

Figure 2: Example rewrite rule for passive normalization

ment is for instance the mechanism of packed rewriting that allows for an efficient representation and processing of ambigous f-structure analyses.

The semantic conversion, as described in (Crouch and King, 2006), is not a priori constrained by a formal apparatus of meaning assembly. The main intuition of the conversion is that the embeddings encoded in the syntactic analysis have to be normalized or reencoded in a way such that they correspond to a semantic embedding. An example rewrite rule which applies to passive f-structure analyses and converts them to an active analysis is given in figure refpassivefig.

In order to be maintainable and extensible, the set of transfer rules producing the semantic representations are organized in a modular way. The main steps of the semantic conversion are given in the following: (i) Flattening of syntax specific f-structure embeddings that don't correspond to semantic embeddings (ii) Canonicalization of grammatical relations (e.g. depassivization) (iii) Marking of items that induce a semantic embedding (which is not encoded in the f-structure) (iv) Linking of f-structure scopes and context of the semantic representation. (v) Removing of f-structure specific features.

An explicitely modular conception of the transfer procedure also facilitates its porting to other languages. Thus, steps 1 and 2 (and partly 3) may be dependent on the language specific f-structure encoding, while the general steps from 3 and 5 don't have to be changed at all when porting the transfer rules to another language. 


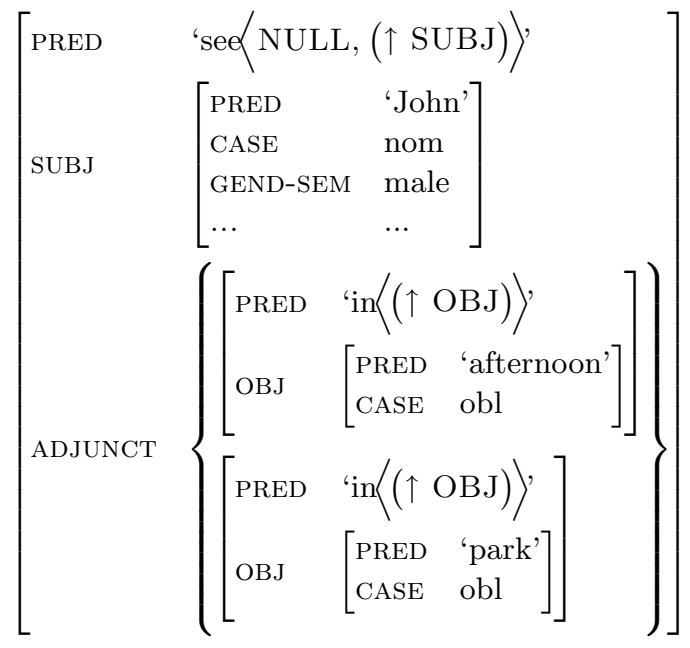

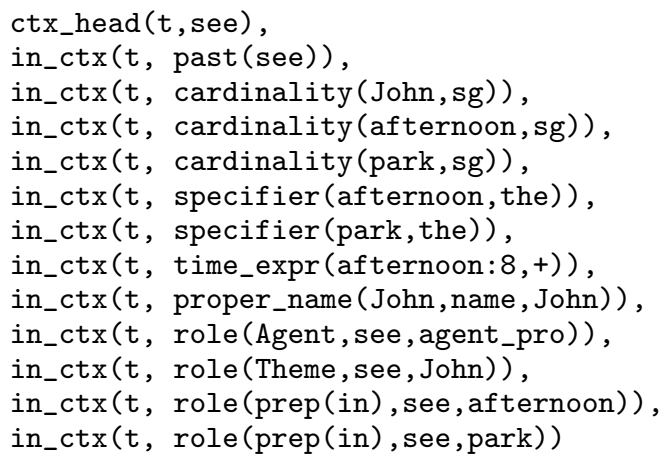

Figure 1: LFG f-structure analysis and corresponding semantic representation

\section{From English to German Semantics}

\subsection{Semantic Grammar Development}

In contrast to the various gold standard treebanks available for the development and evaluation of parsers, gold standards for semantic representations are hardly available. This has a number of methodological implications for "semantic grammar" development. For instance, the authors in (de Paiva and King, 2008) argue for large-scale development of a semantics that is based on an applicationoriented testsuite of entailment pairs instead of sentences and their theoretically correct representations. However, in the context of this work, we didn't focus on a semantic application, but we wanted to assess the portability of the semantic representations to other languages directly. Adopting such a theorydriven perspective on semantic grammar development, the only possibility to account for the accuracy of the semantic construction is to manually inspect the output of the system for a necessarily small set of input sentences.

Moreover, the transfer scenario complicates the assessment of the system's coverage. While in (Bos et al., 2004), the coverage of the meaning construction can be quanti- fied by the number of syntactic analysis that the construction algorithm can process, the transfer conversion will never fail on a given syntactic input. Since the transfer rules just try to match the input, the unmatched features just pass unchanged to the output and will be probably deleted by some of the catchall rules which remove remaining syntactic features in the final step of the conversion. Therefore, manual inspection is necessary to see whether the conversion has processed all the input it was supposed to process.

This limited evaluation scenario entails that the semantics developer has to think hard about defining the set of phenomena he wants to cover and document precisely which type of syntactic phenomena his semantics intends to assign an interpretation to. Therefore, in the rest of this section, we will try to give a concrete overview of the type of phenomena that is covered by the EnglishGerman semantics.

\subsection{A Parallel Testsuite}

In consequence to these considerations on evaluation, a central aspect of our development metholodogy is a testsuite of German sentences which represents the "core semantics" that our systems covers. The multi- 
lingual perspective provided a major orientation for the composition of this testsuite. As our base English semantics implicitely defines a set of core phenomena interpreted by the syntax-semantic interface, we dispose of a set of grammatical f-structure relations that receive a particular semantic representation. Fortunately, the developers of the English semantics had documented many "core" transfer rules (assuring the normalization and context embedding) with example phrases or sentences such that one could easily reconstruct the type of phenomenon each transfer rule was intended to analyze.

On the basis of this system documentation, we first conceived an English testsuite where each sentence contained a construction related to the application of a specific transfer rule. For each of the sentences we selected a German sentence which exhibited the German counterpart of the phenomenon targeted in the English sentence. For instance, if a transfer rule for relative clauses fired on a given English sentence we translated the German sentence such that it contained a relative clause. As most of the test sentences target fairly general phenomena at the syntax-semantic interface (see the next section), there was a parallel German realization of the construction in most of the cases.

In cases where no straightforward parallel realization could be found, we recur to a semantically parallel translation. For instance, the English cleft construction exemplified by the following sentence of our testsuite, does not have a syntactically parallel realization in German. In this case, the sentence was translated by a "semantic" equivalent that emphasizes the oblique argument.

$$
\begin{aligned}
& \text { a. It is to the store that they went. } \\
& \text { b. Zum Markt sind sie gegangen. }
\end{aligned}
$$

During the development process, the testset was further extended. These extensions were due to cases where the English grammar assigns a uniform analysis to some constructions that the German gramamr dis- tinguishes. For instance, while the English grammar encodes oblique arguments the same way it encodes direct objects, the German grammar has a formally slightly different analysis such that rules which fire on obliques in English, don't fire for German input. Now, the final parallel testsuite comprises 200 sentence pairs.

The following enumeration lists the basic morpho-syntactic phenomena covered by our core semantics testsuite.

1. Sentence types (declaratives, interrogatives, quotations etc.)

2. Coordination (of various phrase types)

3. Argument - semantic role mapping, including argument realization normalization (depassivization etc.)

4. Sentential and verbal modification (discursive, propositional, temporal, etc.)

5. Nominal modification (measures, quantifiers, comparatives, etc.)

6. Tense and aspect

7. Appositions and titles

8. Clause-embeddings, relative clauses, gerunds, etc.

\section{Predicative and copula constructions}

\section{Topicalization}

It turns out that the abstract conception of LFG f-structure analysis already assumes a major step towards semantic interpretation. Many global syntactic properties are explicitely represented as feature-value pairs, e.g. features for sentence type, mood, tense and aspect. Moreover, the f-structure already contains many information about e.g. the type of nominal phrases (proper names, quantified phrases etc.) or types of modifiers (e.g. adverb types). Finally, this also justifies our testsuite approach since the range of syntactic variation on this abstract level is much smaller than on the level of word-order. 


\subsection{Parallel Core Semantics}

The English core semantics developped by (Crouch and King, 2006) comprises 798 (ordered!) rewrite rules. As we hypothesized that a major part of the English rules will also apply to German f-structure input, we first copied all English transfer rules to the German semantics and then proceeded by manual error correction: For each German test sentence, we manually checked whether the transfer semantics produce an interpretation of the sentence which is parallel to the English analysis. In case a mismatch was detected, the respective rules where changed or added in the German transfer rule set.

To cover the 200 sentences in our parallel testsuite, 47 rewrite rules had to be changed out of the 798 rules which constitute the core English semantics. Out of these 47 rules, 23 rules relate to real structural differences in the f-structure encoding for German and English. The rest of the modifications is mainly due to renamings of the features or lexical items that are hard-coded in the transfer grammar.

While in a more surface-oriented syntax, it would be hardly possible to design largely parallel syntax-semantic interfaces for the range of phenomena listed in the last section, the surface-independence (and the resulting relative crosslingual generality) of LFG $\mathrm{f}$ structures ensures that a major part of the English core semantics straightforward-ly applies to the German input.

An impressive illustration of the language independence of LFG f-structure analyses in the ParGram grammars is the pair of analyses presented in figure 3, produced by the semantic conversion for the example pair in (3).

$$
\begin{aligned}
& \text { a. Wo hat Tom gestern geschlafen? } \\
& \text { b. Where did Tom sleep yesterday? }
\end{aligned}
$$

The representation for the German sentence was produced by running the English transfer semantics on German syntactic in- put. Although the word-order of English and German questions is governed by distinct syntactic principles, the semantic representation of the German sentence is almost entirely correct since the f-structure analyses abstract from the word-order differences. The only fault in the German representation in 3 is the interpretation of the temporal adverb yesterday - gestern. The transfer rule for temporal verb modification didn't fire because the adverb type features for English and German differ.

\subsection{Discussion: Clause-embeddings and Semantic Fine-graininess}

The crosslinguistic parallelism of the semantics presented in this paper is also due to the relative coarse-grained level of representation that interprets many phenomena prone to subtle crosslingual divergences (e.g. the interpretation of quantifiers or tense and aspect) in terms of conventionalized predications, e.g. the interpretation of tense as past (see) in figure 1. Thus, the real semantic interpretation of these phenomena is deferred to later representation or processing layers, as in this framework, to the definition of entailment relations (Bobrow et al., 2007b). A meaning representation that defers much of the semantic interpretation to the formulation of entailment rules runs the obvious risk of making to few theoretical generalizations which results in very complex entailment rules. This section will briefly illustrate this problem by discussing the representation of clause-embeddings in our semantics.

The various semantic operators defined by the semantic conversion to induce an embedding (see section 2.1) embed a semantic entity of the type context which can be roughly considered as the common semantic type of "proposition". An example for the semantic representation of a clause-embedding verb is given in figure 4 .

For many semantic applications, such embedded contexts are of particular interest since they often express propositions to 


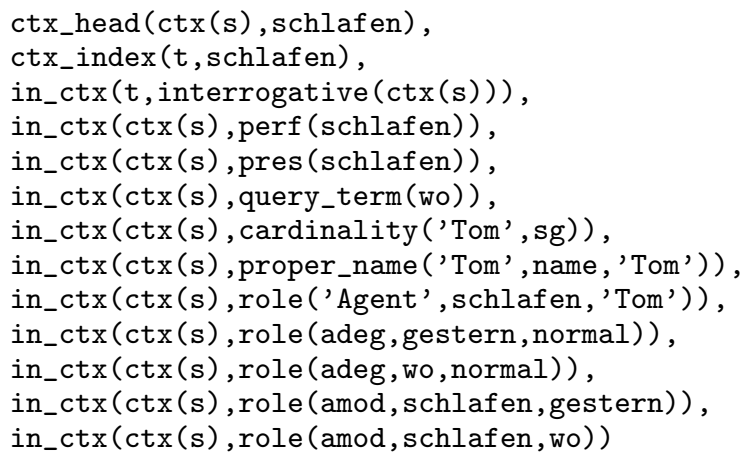

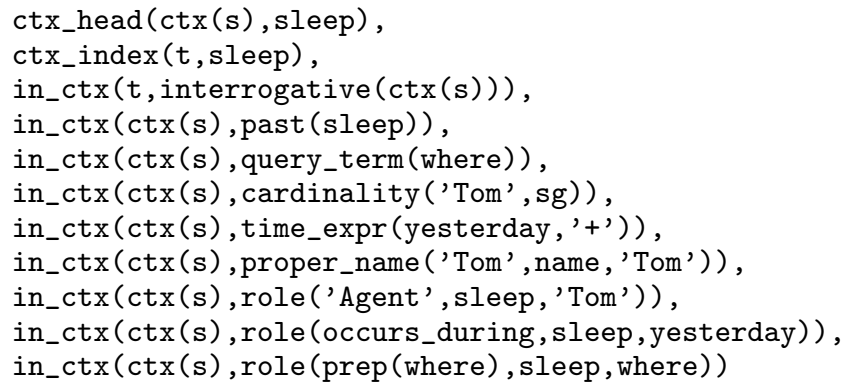

Figure 3: Parallel semantic analyses for the sentence pair given in example (3)

whom the speaker is not committed to, i.e. which aren't veridical. In our system, the veridicality inferences that these embeddings exhibit are computed by further knowledge representation modules that explicitely represent the speaker commitment of a context (Bobrow et al., 2007b). Concerning the complements of clause-embedding verbs, these inferences are modelled via a lexical verb classification that basically distinguishes implicatives (manage to TRUE - don't manage to FALSE) and factives (know that TRUE don't know that TRUE) (Nairn et al., 2006). Veridicality entailments of sentential complements are treated as a interaction of the lexical class of the subordinating verb and the polarity of the context.

(4) Tom glaubt, dass der Nachbar ihn nicht erkannt hat.

'Tom believes that the neighbour didn't recognize him.'

This account of clause-embeddings - a unified semantic representation and a lexical entailment classification - generalizes and probably simplifies too much the various theoretical insights into the semantics of complementation. In the formal semantics literature, various theories opt for a semantic representation that assumes several types of abstract semantic entities (e.g. events (Parsons, 1990), situations (Barwise and Perry, 1999) or other, very fine-grained categories (Asher,
1993) ). In terms of entailment, the typological literature reports crosslingually relatively stable distinctions of types of complements according to the semantic relations the matrix verbs have to their complement (Givon, 1990). For instance, while in example (5), the infinite complement has causal, temporal and spatial relations to the matrix event, there is no such inferential relation between matrix and complement in example (4) .

(5) Seine Freundin brachte ihn dazu, ein Haus zu bauen.

His girlfriend made him build a house.

Moreover, the semantics of clauseembedding verbs shows subtle distinctions with resepct to other linguistic features (apart from the polarity of the context) that can trigger a particular speaker commitment. For instance, in languages that have a morphological aspect marking (like Frensh, in the following example), the following aspectually motivated entailments can be observed (see (Bhatt, 2006)):

(6) Jean pouvait soulever cette table, mais il ne l'a pas fait.

'Jean was able.IMP to lift this table, but he didn't do it.'

(7) Jean a pu soulever cette table, \#mais il ne l'a pas fait.

'Jean was able.PERF to lift this table,\#but he didn't do it.'

In sentence (6), the imperfect aspect 
causes the modality of the complement such that it is not necessarily true, while in sentence (7), the embedded clause is necessarily true due to the perfective aspect of the clause-embedding verb. This aspectual matrix-com-plement relation is however only observable for certain types of modality or clause-embedding verbs and has no clear semantic parallel in other languages that don't have aspectual marking.

For another type of clause-embedding verbs, called epistemic verbs, the recent formal semantics literature discusses many examples where the lexical neutral entailment class is overriden by pragmatic interpretation constraints that cause the embedded complement to be interpreted as true although the embedding operator does not entail the veridicality of its complement (Simons, 2006; von Fintel and Gillies, 2007). As an example, consider the following text - hypothesis pair annotated as a valid entailment in the Pascal RTE 3 set altough the hypothesis clearly refers to an embedded proposition in the given text.

Between March and June, scientific observers say, up to 300,000 seals are killed. In Canada, seal-hunting means jobs, but opponents say it is vicious and endangers the species, also threatened by global warming.

(9) Hunting endangers seal species. FOLLOWS (RTE3 ID:225)

Such examples suggest that entailments concern various aspects of the meaning of a sentence or proposition, thus, not only its veridicality but also its temporal properties, informations about involved agents, space and time. These properties are clearly related to the semantic type of the embedded clause.

Purely lexical entailment rules for clauseem-bedding operators will be very hard to formulate in the light of the complex interaction of the various linguistic parameters. These considerations reveal a general trade-off between a representation that gen-

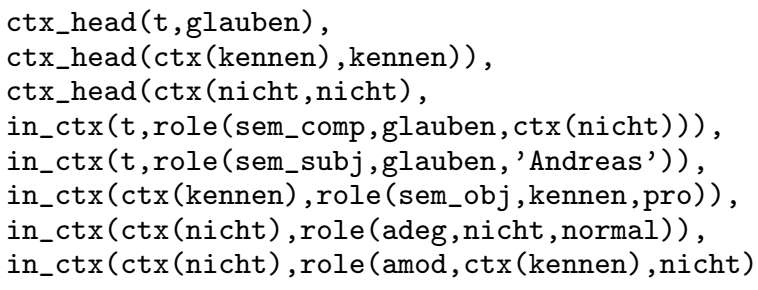

Figure 4: Example representation for context embeddings, sentence (4)

eralizes over many (purely) theoretical and crosslingual subtleties and a representation that does not capture certain generalizations which would lead to a more linguistically informed account of entailment relations. Future work on the semantics presented in this paper will have to take such tensions into account and think about the general goals and applications of the semantic representation.

\section{Conclusion}

This work amply illustrates the positive implications of crosslinguistic, parallely designed resources for large-scale linguistic engineering. Due to the abstract f-structure layer in LFG syntax and its parallel implementation in the ParGram project, further resources that build on f-structure representations can be very easily ported to other languages. Future research will have to investigate to what extent this also applies to more distant languages, like Urdu and English for instance.

The paper also discussed some problematic aspects of the development of a large-scale semantic system. The crosslingual development perspective allowed us to define a set of core semantic phenomena covered by the representation. However, from a formal semantic view point, the simplifying representation obstructs potential crosslingual differences in semantic interpretation. Future research still has to be conducted to develop a more general development and evaluation methodology for the representation of meaning. 


\section{References}

Nicholas Asher. 1993. Reference to Abstract Objects in Discourse. Studies in Linguistics and Philosophy. Kluwer Academic Publishers.

Jon Barwise and John Perry. 1999. Situations and Attitudes. CSLI Publications.

Rajesh Bhatt, 2006. Covert Modality in Non-finite Contexts, volume 8 of Interface Explorations, chapter Ability modals and their actuality entailments. Mouton de Gruyter.

Daniel G. Bobrow, Bob Cheslow, Cleo Condoravdi, Lauri Karttunen, Tracy Holloway King, Rowan Nairn, Valeria de Paiva, Charlotte Price, and Annie Zaenen. 2007a. PARC's Bridge question answering system. In Tracy Holloway King and Emily M. Bender, editors, Proceedings of the GEAF (Grammar Engineering Across Frameworks) 2007 Workshop, pages 13-15.

Daniel G. Bobrow, Bob Cheslow, Cleo Condoravdi, Lauri Karttunen, Tracy Holloway King, Rowan Nairn, Valeria de Paiva, Charlotte Price, and Annie Zaenen. 2007b. Precision-focused textual inference. In ACL-PASCAL Workshop on Textual Entailment and Paraphrasing, pages $28-29$.

Johan Bos, Stephen Clark, Mark Steedman, James R. Curran, and Julia Hockenmaier. 2004. Widecoverage semantic representations from a CCG parser. In COLING '04: Proceedings of the 20th international conference on Computational Linguistics, page 1240, Morristown, NJ, USA. Association for Computational Linguistics.

Joan Bresnan. 2000. Lexical-Functional Syntax. Blackwell, Oxford.

Miriam Butt and Tracy Holloway King. 2007. XLE and XFR: A Grammar Development Platform with a Parser/Generator and Rewrite System. In International Conference on Natural Language Processing (ICON) Tutorial.

Miriam Butt, Helge Dyvik, Tracy Holloway King, Hiroshi Masuichi, and Christian Rohrer. 2002. The Parallel Grammar Project.

Richard Crouch and Tracy Holloway King. 2006. Semantics via F-Structure Rewriting. In Miriam Butt and Tracy Holloway King, editors, Proceedings of the LFG06 Conference.

Dick Crouch, Mary Dalrymple, Tracy King, John Maxwell, and Paula Newman, 2006. XLE Documentation.

Dick Crouch. 1995. Packed Rewriting for Mapping Semantics to KR. In Proceedings of the International Workshop on Computational Semantics.
Mary Dalrymple. 1999. Semantics and Syntax in Lexical Functional Grammar: The Resource Logic Approach. MIT Press, Cambridge, Mass.

Valeria de Paiva and Tracy Holloway King. 2008. Designing testsuites for grammar-based systems in applications. In Proc. of the COLING GEAF Workshop 2008.

Anette Frank. 1999. From Parallel Grammar Development towards Machine Translation (shortened version). In Miriam Butt and Tracy Holloway King, editors, Proceedings of the LFG-99 Conference, CSLI Online Publications, University of Manchester. Section 4 of: Miriam Butt and Stefanie Dipper and Anette Frank and Tracy Holloway King.

Talmy Givon. 1990. Syntax, volume 2. Benjamins.

Roger Kim, Mary Dalrymple, Ronald M. Kaplan, Tracy Holloway King, Hiroshi Masuichi, and Tomoko Ohkuma. 2003. Multilingual Grammar Development via Grammar Porting. . In ESSLLI 2003 Workshop on Ideas and Strategies for Multilingual Grammar Development.

Rowan Nairn, Cleo Condoravdi, and Lauri Karttunen. 2006. Computing relative polarity for textual inference. In Inference in Computational Semantics (ICoS-5).

Terence Parsons. 1990. Events in the semantics of English, volume 19 of Current studies in linguistics series ; 19. MIT Pr., Cambridge, Mass. [u.a.].

Mandy Simons. 2006. Observations on embedding verbs, evidentiality, and presupposition. Lingua.

Kathrin Spreyer and Anette Frank. 2005. The TIGER 700 RMRS Bank: RMRS Construction from Dependencies. In Proceedings of LINC 2005, pages $1-10$.

Hiroshi Umemoto. 2006. Implementing a Japanese Semantic Parser Based on Glue Approach. In Proceedings of The 20th Pacific Asia Conference on Language, Information and Computation.

Kai von Fintel and Anthony S. Gillies. 2007. An opinionated guide to epistemic modality. In Tamar Gendler Szabo and John Hawthorne, editors, Oxford Studies in Epistomology, Vol. 2. Oxford University Press. 